\title{
Recognition of human activity based on sparse data collected from smartphone sensors*
}

\author{
Joao Figueiredo $^{1,3}$, Goncalo Gordalina ${ }^{2}$, Pedro Correia ${ }^{1,3,7}$, Gabriel Pires ${ }^{1,3,8}$, Luis Oliveira ${ }^{1,3,7}$, \\ Ricardo Martinho ${ }^{2,5}$, Rui Rijo ${ }^{2,5,6,9}$, Pedro Assuncao ${ }^{2,7}$, Alexandra Seco ${ }^{2}$ and Rui Fonseca-Pinto ${ }^{2,4,7}$
}

\begin{abstract}
This paper proposes a method of human activity monitoring based on the regular use of sparse acceleration data and GPS positioning collected during smartphone daily utilization. The application addresses, in particular, the elderly population with regular activity patterns associated with daily routines. The approach is based on the clustering of acceleration and GPS data to characterize the user's pattern activity and localization for a given period. The current activity pattern is compared to the one obtained by the learned data patterns, generating alarms of abnormal activity and unusual location. The obtained results allow to consider that the usage of the proposed method in real environments can be beneficial for activity monitoring without using complex sensor networks.
\end{abstract}

\section{INTRODUCTION}

The use of wearable inertial sensors for human activity monitoring has gained popularity due to its versatility and energy saving aspects when compared with traditional approaches using external sensors [1]. In particular, several applications have been proposed for remote monitoring of vital signs and human activity classification [2]. On the other hand, the increasingly widespread use of smartphones opened the possibility of using a wide range of sensors embedded in the phone to develop human activity monitoring applications, without needing additional devices.

Although daily activities and behaviours vary dependently on external factors, there are some regular activity patterns associated with everyday practices. This is especially true in the elderly population, even more for those that have functional limitations and reduced mobility. These patterns can be analysed by individual tasks, or by a group of tasks. By aggregating and extracting attributes associated with specific tasks, it is possible to monitor patterns of daily activity and

*This work has been financially supported by the IC\&DT Project MOVIDA: SAICT-POL/23878/2016 and Project VITASENIOR-MT: SAICT-POL/23659/2016 with FEDER funding through programs CENTRO2020 and FCT.

${ }^{1}$ J.Figueiredo, P. Correia (pcorreia@ipt.pt), L. Oliveira and G. Pires are with School of Technology, Polytechnic Institute of Tomar, Tomar, Portugal.

${ }^{2}$ G. Gordalina, R. Martinho, R. Rijo, P. Assuncao, A. Seco and R. Fonseca-Pinto are with School of Technology and Management, Polytechnic Institute of Leiria, Leiria, Portugal.

${ }^{3}$ J.Figueiredo, P. Correia, L. Oliveira and G. Pires are with VITA Lab./Smart Cities Research Center(C2I2), Tomar, Portugal.

${ }^{4} \mathrm{R}$. Fonseca-Pinto is with CiTechCare - Center for Innovative Care and Health Technology, Polytechnic Institute of Leiria, Leiria, Portugal.

${ }^{5}$ R. Martinho and R. Rijo are with Centre for Research in Health Technologies and Information Systems (CINTESIS), University of Porto, Porto, Portugal.

${ }^{6}$ R. Rijo is with Health Intelligence Laboratory, Faculty of Medicine of the University of Sao Paulo, Ribeirao Preto/Sao Paulo, Brazil.

${ }^{7}$ P. Assuncao, R. Fonseca-Pinto, P. Correia and L. Oliveira are with Instituto de Telecomunicacoes, Portugal.

${ }^{8}$ G. Pires is with Institute of Systems and Robotics (ISR-UC), University of Coimbra, Coimbra, Portugal.

${ }^{9} \mathrm{R}$. Rijo is with Institute for Systems Engineering and Computers at Coimbra (INESC Coimbra), University of Coimbra, Coimbra, Portugal. detect changes in these patterns that can be used to generate several types of alarms for the user himself, or to a caregiver. Usually, automatic human activity recognition uses a wide range of sensors, which can be placed in several parts of the body to infer specific human activities such as, walking, running or stair climbing [3]. However, this approach can be used in a laboratory controlled environment, but it can not be used in real daily activities without disturbing the regular exercise. Alternatively, other types of sensors can be placed in different parts of the living house to recognize domestic activities like cooking, sleeping or watching TV [4]. Generally, we can say that the higher the number of sensors used, the more accurate are the classification results.

Smartphones have a set of embedded sensors available, such as accelerometers, gyroscopes, magnetometers, light sensors, cameras, microphones, GPS, and others. Since smartphones are widely available for a large group of the population, it is a very interesting device to be used as a support for monitoring applications of human activity. Nevertheless, it has also some limitations that are related to the type of use, the position, and orientation of the device, and battery autonomy. For example, the user can spend more or less time using the smartphone, and the smartphone can be placed on the pocket or at hand, or just on the table.

Human activity classification is usually based on supervised approaches, that compare current activity with training sets labeled with known activities. However, this labeling is hard to obtain and is usually performed in a controlled environment, which can be far from the real environment. Some automatic labeling methods have been proposed to avoid the manual methods [5]. On the other hand, unsupervised classification using clustering and deep learning methods were introduced to firstly identify patterns from data and then trying to label specific activities [6], [7]. A distinct approach is to find patterns without knowing the activity, and then use trained data to understand whether an activity is regular or not.

This paper proposes an approach for human activity monitoring based on the regular use of acceleration data and GPS positioning collected during smartphone daily utilization, focusing on the elderly population that usually has the same daily routines, in one or two distinct places. For instance, they are considered people that live alone at home, in a senior residence, or spend periods of the day in a caring center.

Using sparse collected data, i.e. with a small number of sensors, reduced sampling ratio and only supported by a free handle device, the monitoring aims to find regular patterns, without knowing the specific activities, and then generate alarms when an activity has a different pattern. This approach opens new research challenges, since is not based on specific labeling, the available number of sensors is reduced, and is 
dependent on the normal use of the smartphone, increasing the uncertainty of the classification.

\section{TRACKING Human ACtivity}

\section{A. MOVIDA.DOMUS Platform}

The MOVIDA.domus [8] is a human activity monitoring platform comprising four modules: Mobile App; Web Platform; Cloud services; web service-based Application Programming Interface (API) and Artificial Intelligence (AI) server. The mobile application gathers the acceleration data in its different axes and GPS data. Using a service-based API, the AI server, regularly collect the data of each user and perform data analysis and activity monitoring by finding the activity patterns to detect abnormal daily routines. Depending on the similarity between activity patterns, several types of alarms are generated, to be sent to the user and caregivers, namely, absent activity, abnormal activity, and unusual location.

\section{B. Data Gathering}

The sampling frequency of the acceleration and GPS data is $1 \mathrm{~Hz}$. However, for the GPS data, the value is only considered when the position is different from the previous one, in 1 meter. In order to save energy, the data is sent to the server through the service-based API, every 8 minutes.

Data acquisition for processing by the AI server is done using native MATLAB functions, webread \& webwrite, to connect to the service-based API. The API responds with JSON, which MATLAB converts to struct type. The data is then parsed, and finally, the variables containing the GPS and acceleration data are saved into files.

\section{PAtTern Activity Recognition}

Figure 1 shows the pipeline of the pattern activity recognition system. The activity patterns are defined using unsupervised clustering. The clustering for both acceleration and GPS values is done using k-means, where the initial values are chosen using k-means++ [9]. The number of clusters for acceleration is defined automatically using the DaviesBouldin index (DBI) [9] or the Calinski \& Harabasz $(\mathrm{CH})$ criterion [9]. In the case of GPS, the final number of clusters is chosen based on a distance threshold.

\section{A. Clustering of acceleration}

To characterize the human activity in a given period, the clustering step occurs only when a sufficient number of points is gathered (e.g. 200,000 points). After the first clustering, a sliding window with $50 \%$ overlap is used to adapt the cluster learning (i.e. clustering is repeated after 100,000 new points). Using acceleration data, two clustering methods are used: 1) magnitude-based clustering that uses the values of the norm of acceleration and 2) feature-based clustering, that uses several features taken from acceleration axes combined with Principal Component Analysis (PCA) before clustering. Finally, the test values are compared with the obtained regular activity patterns using 3600 points, finding if the test activity data matches or not with the learned data pattern.

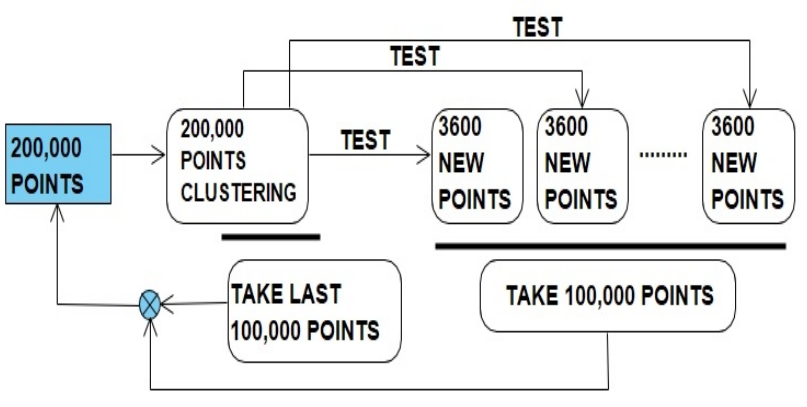

Fig. 1. Clustering and comparison mechanism (acceleration).

1) Method 1 - Magnitude-based clustering: This method uses the raw 3 -axis acceleration values $(x, y, z)$. The magnitude of acceleration $\left(a=\sqrt{x^{2}+y^{2}+z^{2}}\right)$ is calculated, subtracted by 9.8 and then, passed through a simple moving average (SMA) filter, in order to smooth the data. The filter's output data is then used in the clustering. The method is described in Algorithm 1.

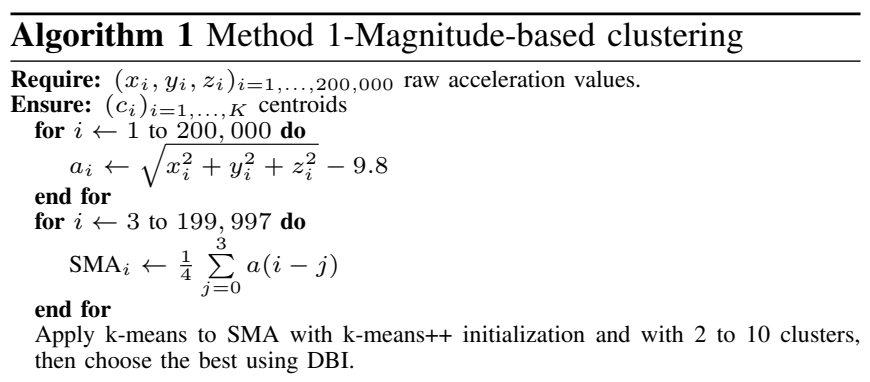

Figure 2 presents an example of clustering results from training data, where a statistical difference between the clusters can be observed.
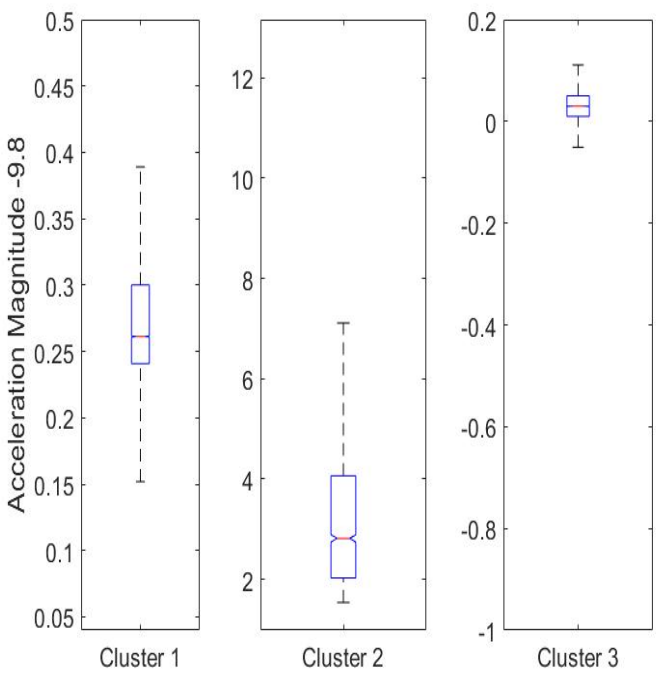

Fig. 2. Magnitude-based clustering.

2) Method 2 - Feature-based clustering: The featurebased clustering method is described in Algorithm 2. The 
magnitude of acceleration and individual components are used, and then, an SMA filter is applied to the four signals. After that, several features are extracted using a 12 second window. The extracted features for each acceleration axis and corresponding magnitude are presented in Table I. After feature extraction, 58 features in total are obtained. Principal component analysis (PCA) is applied to reduce the dimensionality to two. The data is then clustered.

TABLE I

FEATURES EXTRACTED

\begin{tabular}{|c|c|}
\hline Feature & Feature dimension (per signal) \\
\hline \multicolumn{2}{|c|}{ Statistical } \\
\hline Mean & 1 \\
\hline Standard Deviation & 1 \\
\hline Interquartile Range & 1 \\
\hline Kurtosis & 1 \\
\hline Skewness & 1 \\
\hline \multicolumn{2}{|c|}{ Time Domain } \\
\hline Root Mean Square & 1 \\
\hline Zero Crossing Rate & 1 \\
\hline Autocorrelation & 1 \\
\hline Pairwise Correlation & 3 (6 in total) \\
\hline Median Absolute Deviation & 1 \\
\hline \multicolumn{2}{|c|}{ Frequency Domain } \\
\hline Median Frequency & 1 \\
\hline Maximum Frequency & 1 \\
\hline Fundamental Frequency & 1 \\
\hline Total Energy & 1 \\
\hline
\end{tabular}

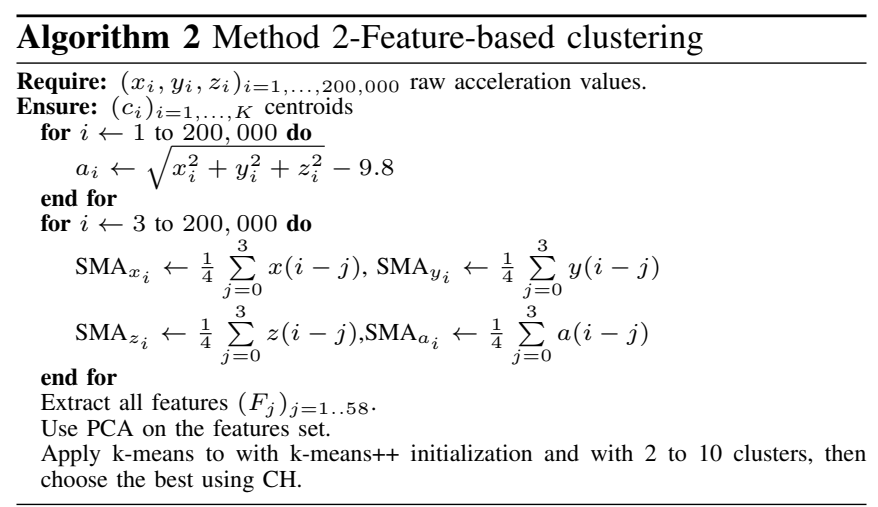

Figure 3 shows the clustering results using feature-based method. Several clusters are generated and well defined, giving the ability to detect abnormal activity.

\section{B. Clustering of GPS}

There is a GPS clustering process for each hour of the day (24 clusters). Each cluster uses 300,000 GPS points with 50\% overlap. Therefore, data is first separated by hour. For each hour the clustering begins, first with one cluster, then two, and so on, until $90 \%$ of the data is located at a 200 meters of distance from at least one of the centroids. The clustering of GPS is explained in Algorithm 3.

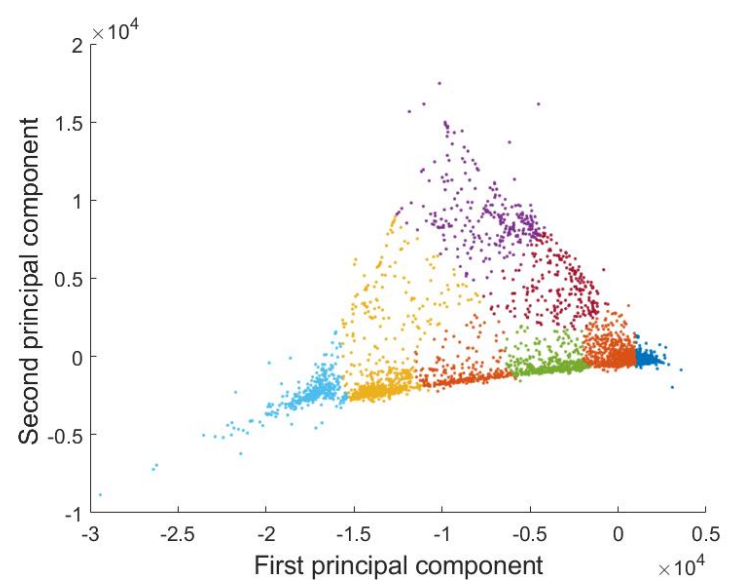

Fig. 3. Feature-based clustering.

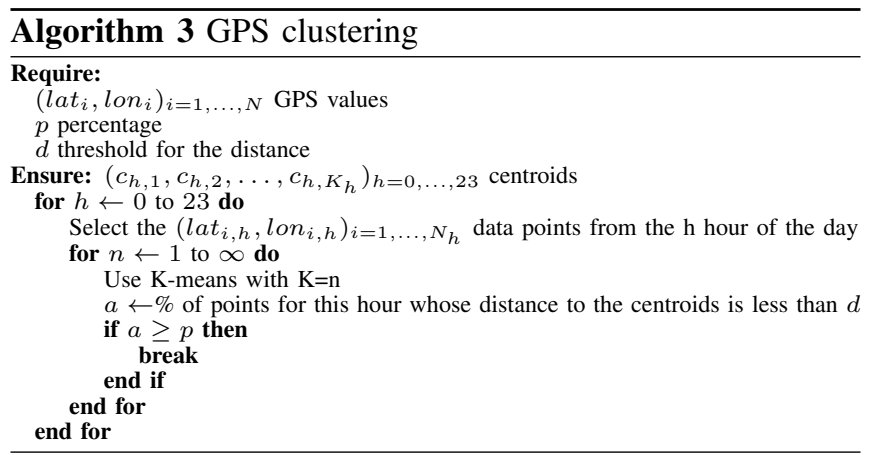

\section{Alarm Generation Algorithms}

Clustering methods are used to infer if current activity is classified as normal or abnormal. There are three types of algorithms for abnormal activity detection. We aim to compare them and determine the one with the best results. The algorithms make use of the clustering to perform a comparison between cluster data and newly gathered values. If the output of the comparator is higher than a given threshold experimentally obtained, an alarm is sent. The comparison is done every new 3600 points.

\section{A. Abnormal Activity Algorithms}

1) Algorithm 1 (Magnitude-based clustering): This algorithm makes use of the magnitude-based clustering. The algorithm checks which new points belong to each centroid. For every centroid, the ratio of the new points that belong to it are subtracted with the corresponding ratio calculated for the points used in the clustering. The differences for each centroid are taken, and its absolute value added to a total. If the total is higher than one, an alarm is sent.

2) Algorithm 2 (PCA): This algorithm takes the last 200,000 magnitude values, together with their time stamp. For every value, the algorithm gets the hour of the day at which it was gathered. After that PCA is performed. The same is done to the 3600 new points. Then, the average of the distance between the new points to the two PCA components (considered as axis) is used in the comparison. If that value is bigger than 1.5, an alarm is sent. 
3) Algorithm 3 (Feature-based clustering): This algorithm makes use of the feature-based clustering algorithm. It takes the new points and performs an equivalent testing clustering (the same number of clusters, the same clustering method, applied to the same features). Then, for each of the testing centroids, calculates the minimal distance to the centroids of the training clustering, doing its average. If that average divided by $1 \times 10^{5}$ is bigger than 3 , an alarm is sent.

\section{B. Abnormal Position Algorithm}

This method intends to be used to generate a notification to the caregiver, whenever some user moves away from usual locations. The proposed algorithm uses clustering of outdoor GPS values to infer the usual locations. The method takes a newly gathered GPS position and checks if any of the clustering centroids are at an acceptable distance from it (200 meters). If not, an alarm is sent. Since indoor positioning is hard to obtain, the method only considers outdoor GPS values. For indoor localization, other contextaware approaches should be considered.

\section{ANALYSIS AND RESUlTS}

For comparison of the different methods, the data of a volunteer was used, specifically the acceleration values corresponding to two weeks of regular use of the smartphone. The records of the daily routines were performed by the volunteer to test which of the proposed algorithms had a higher sensitivity level. After the first 200,000 points required for the first clustering, the user performed different routines as a way to trigger a routine alarm.

\section{A. Abnormal Activity Algorithms}

Considering the volunteer data used to test the algorithms, 34 comparisons and alarm decisions were performed. An abnormal activity was identified by the volunteer during the time corresponding to the comparisons 9 and 10. In Figure 4 , the algorithm outputs can be observed, and some visual similarity between the data can be observed.

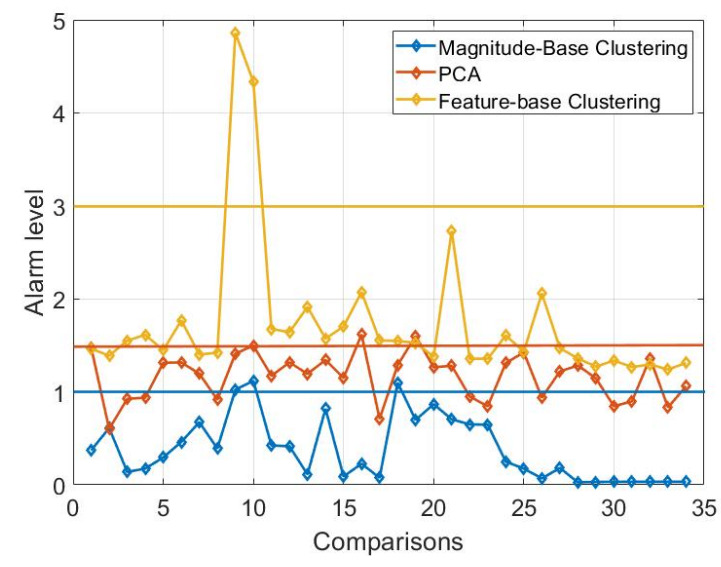

Fig. 4. Abnormal activity detection.

1) Magnitude-based clustering: This algorithm generates 3 alarms, 2 at comparisons 9 and 10, as expected. Another alarm was generated during comparison 18 (Figure 4) which was unexpected, according to the volunteers feedback . This false positive requires further study, to see if it is related with the users smartphone use (ex: a long call).
2) PCA: In this case, only an alarm in position 10 was generated. Additionally, 2 false positives were identified through the users feedback. The values themselves show a lot of variation, considering their small amplitude.

3) Feature-based clustering: This is the method with better results, generating 2 and only 2 alarms at positions 9 and 10, as expected. The algorithm presents a reasonable range of values, resulting in accurate results compared with previous methods. However, further study is required, with different users scenarios and activities, for better validation.

\section{B. Abnormal Position Algorithm}

The GPS results were obtained using feedback from users. Generally, the GPS clustering algorithm converge to the usual locations. In hours where activities are well defined, the obtained GPS centroids converges to the regular positions reported by the user. As it would be expected, the number of clusters is increased when position variability is high for a given hour.

\section{CONCLUSIONS}

This work presents a human monitoring approach using collected acceleration and GPS data of a smartphone. Two clustering methods are compared, namely clustering using acceleration data and other using a set of features based on acceleration signals and its combination with PCA, to find regular patterns of activity. Abnormal activity alarms are generated when compared with current data. Also, regular outdoor positioning patterns are made based on GPS data. The presented results show the effectiveness of the methods to identify an abnormal activity based on trained data, to generate correspondent alarms. In future work, clustering will include time and localization features to improve the accuracy of recognition of the regular activity and consequently the alarm generation.

\section{REFERENCES}

[1] Oscar D. Lara and Miguel A. Labrador, A Survey on Human Activity Recognition using Wearable Sensors, IEEE Communications Surveys \& Tutorials, Vol. 15, No.3, pp. 1192-1209, Third Quarter 2013.

[2] Muhammad Awais, Luca Palmerini, Alan K. Bourke, Espen A. F. Ihlen, Jorunn L. Helbostad and Lorenzo Chiari, Performance Evaluation of State of the Art Systems for Physical Activity Classification of Older Subjects Using Inertial Sensors in a Real Life Scenario: A Benchmark Study, in Sensors, vol. 16(12), 2105, 2016.

[3] D. De and P. Bharti and S. K. Das and S. Chellappan, Multimodal Wearable Sensing for Fine-Grained Activity Recognition in Healthcare, IEEE Internet Computing, vol. 19, No.5, pp. 26-35, Sept.-Oct., 2015 .

[4] Shumei Zhang, Paul McCullagh, Huiru Zheng, and Chris Nugent, Situation Awareness Inferred From Posture Transition and Location Derived From Smartphone and Smart home Sensors, IEEE Transactions On Human-Machine Systems, Vol.47 No.6,pp. 814-821, 2017.

[5] Federico Cruciani, Ian Cleland, Chris Nugent, Paul McCullagh, Kre Synnes and Josef Hallberg, Automatic Annotation for Human Activity Recognition in Free Living Using a Smartphone, in Sensors, vol. 18(7), 2203, 2018.

[6] Yongjin Kwon, Kyuchang Kang and Changseok Bae, Unsupervised learning for human activity recognition using smartphone sensors, Expert Systems with Applications, Vol. 41, No. 14, pp.6067-6074, 2014.

[7] Andrey Ignatov, Real-time human activity recognition from accelerometer data using Convolutional Neural Networks, Applied Soft Computing, Vol.62, pp. 915922, 2018.

[8] Goncalo Gordalina, Joao Figueiredo, Ricardo Martinho, Rui Rijo, Pedro Correia, Pedro Assuncao, Alexandra Seco, Gabriel Pires, Luis Oliveira, Rui Fonseca-Pinto, Tracking human routines towards adaptive monitoring: the MOVIDA.domus platform, Procedia Computer Science, vol.138, pp. 41-48, 2018.

[9] Aggarwal, Charu C. and Reddy, Chandan K., 2013, Data Clustering Algorithms and Applications, CRC Press, Minneapolis, 652 p. 